\title{
Le Pâturage, C'est toute une science !
}

Savoirs écologiques sur la neige et représentation samie du pâturage

"Pasture, a science in its own right! " Ecological knowledge about snow and the Sami representation of grazing

\section{Samuel Roturier et Marie Roué}

\section{OpenEdition \\ Journals}

Édition électronique

URL : https://journals.openedition.org/tc/7413

DOI : $10.4000 /$ tc. 7413

ISBN : 0248-6016

ISSN : 1952-420X

Éditeur

Éditions de l'EHESS

\section{Édition imprimée}

Date de publication : 1 mai 2015

Pagination : 92-109

ISBN : 9782713224874

ISSN : 0248-6016

\section{Référence électronique}

Samuel Roturier et Marie Roué, « Le Pâturage, C'est toute une science! », Techniques \& Culture [En ligne], 63 | 2015, mis en ligne le 26 décembre 2018, consulté le 29 septembre 2022. URL : http:// journals.openedition.org/tc/7413; DOI : https://doi.org/10.4000/tc.7413 


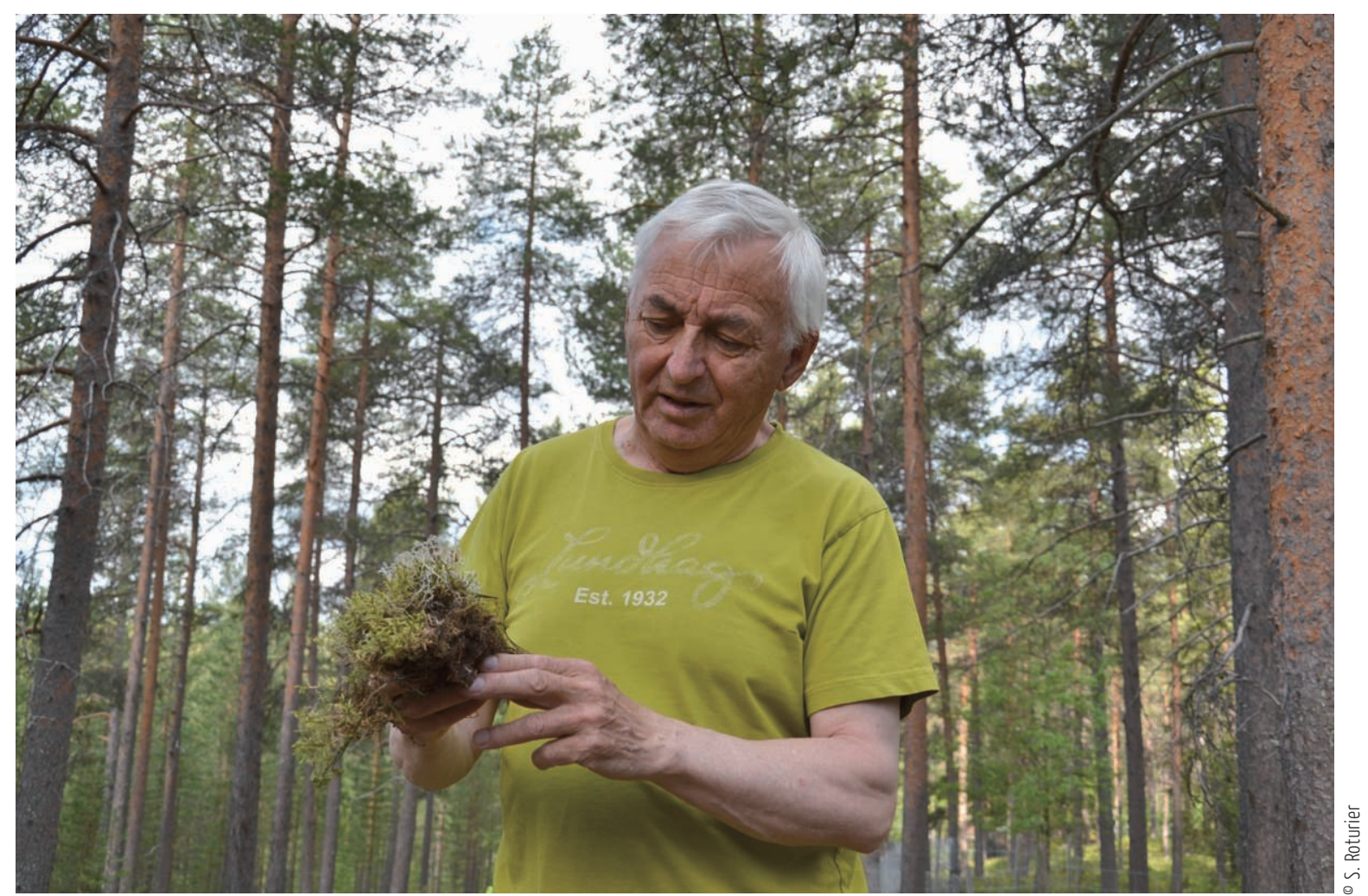




\section{LE PÂTURAGE, C'EST TOUTE UNE SCIENCE !}

\section{Savoirs écologiques sur la neige et représentation samie du pâturage}

« Le pâturage, c'est toute une science en fait ! ». En concluant ainsi sa réponse à une question sur le pâturage hivernal, cet éleveur de renne sami ${ }^{1}$ soulignait la complexité du sujet et l'étendue des savoirs mobilisés pour y répondre. C'est dans le cadre d'un programme de recherche visant à restaurer ${ }^{2}$ les landes à lichen détruites par les aménagements forestiers que nous nous sommes intéressés aux pâturages des rennes. En Suède boréale, l'exploitation forestière a été la dernière des grandes industries basées sur l'exploitation des ressources naturelles, après l'agriculture (dès le XVII ${ }^{e}$ siècle), l'exploitation minière (depuis le xvIII ${ }^{e}$ siècle), la production hydroélectrique (au début du xx ${ }^{e}$ siècle), à coloniser ce vaste territoire habité et exploité par les Samis depuis plusieurs millénaires. Éleveurs samis et forestiers suédois partagent donc aujourd'hui le même territoire, les premiers en hiver pour faire pâturer leurs rennes, les seconds pour exploiter la forêt. Dans la seconde moitié du $\mathrm{xx}^{\mathrm{e}}$ siècle, l'industrie forestière suédoise s'est engagée dans la modernisation et la mécanisation, modifiant profondément les dynamiques et les structures des communautés végétales de la forêt boréale (Esseen \& al., 1997). L'introduction de la coupe à blanc, la fertilisation, la régénération artificielle des peuplements, la construction de pistes forestières, etc., ont ainsi entraîné, et entraînent toujours, des dégradations, voire la destruction de pâturages pour les éleveurs qui, si elles ne sont pas irréversibles, peuvent durer plusieurs décennies. Ces pratiques sont une des causes du conflit entre éleveurs et forestiers qui dure maintenant depuis plus d'un demi-siècle (Roué, 2012). Au début des années 2000 un programme de recherche sur la restauration des pâturages des rennes a ainsi vu le jour à l'initiative de gestionnaires et d'universitaires forestiers. En s'appuyant sur l'écologie et les sciences forestières, ce programme visait d'abord à apporter des

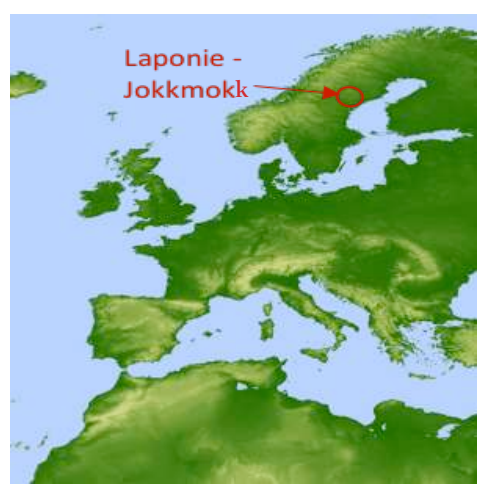



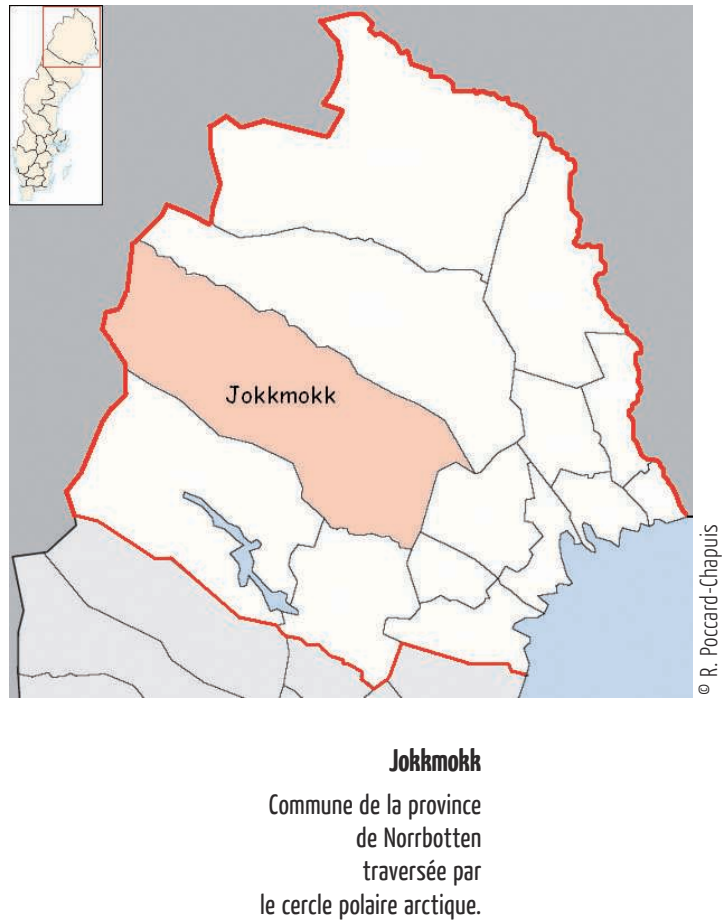

solutions techniques (Roturier, 2009). En mobilisant l'anthropologie, notre collaboration interdisciplinaire a également permis de mettre en lumière les savoirs écologiques samis et leur «science du pâturage ».

Depuis 2008 nous menons un travail de terrain dans la région de Jokkmokk au cours duquel nous avons rencontré, accompagné, interviewé, organisé des ateliers avec une soixantaine d'éleveurs de la région. Ces éleveurs, en général des hommes, ont entre 20 et 70 ans et travaillent avec les rennes depuis l'enfance. Ils peuvent exercer une autre activité, souvent saisonnière, mais l'élevage est leur source principale de revenu. Sur la base de cette recherche de terrain, nous nous proposons d'exposer une partie des savoirs et savoir-faire des éleveurs samis sur le pâturage hivernal des rennes à travers deux questions qui seront développées successivement : Qu'est-ce que le pâturage pour les éleveurs de rennes et dans quelle mesure leur représentation est-elle compatible avec celle des forestiers? Comment le pâturage permet-il aux éleveurs de contrôler les animaux et que traduit-il de leur relation avec les rennes?

L'élevage du renne en Laponie n'est pas inconnu des lecteurs français, particulièrement dans le champ des sciences humaines et sociales où plusieurs anthropologues ont mené des travaux sur cette forme de pastoralisme unique en Europe (Manker, 1954; Paine, 1964 ; Ingold, 1976 ; Delaporte \& Roué, 1986). Il l'est peut-être moins en revanche au sein de la communauté des « pastoralistes » et des zootechniciens. C'est pourquoi au préalable, nous présenterons au lecteur quatre particularités de l'élevage du renne qui tiennent d'une part au contexte autochtone dans lequel s'exerce cette activité, d'autre part au caractère transhumant de l'élevage et à la domestication très particulière du renne, et enfin à la nature même de la ressource pâturée, les lichens, qui permettent aux rennes de survivre à l'hiver boréal ${ }^{3}$.

\section{L'élevage du renne en Laponie suédoise}

\section{Les Samis, peuple autochtone de Fennoscandie}

Les Samis occupent le Nord des pays scandinaves et la péninsule de Kola en Russie. La Laponie est une région interfrontalière définie par son peuplement. On parle ainsi de Laponie norvégienne, finlandaise, suédoise ou encore russe. Dans ces différents pays les Samis partagent une langue, une culture commune et un animal, le renne (Rangifer tarandus tarandus). Chassé, apprivoisé puis élevé, le renne occupe une place importante dans la culture samie (alimentation, vêtements, artisanat). En Suède, l'élevage du renne est associé au peuple sami puisqu'il leur est réservé (ce n'est pas le cas en Finlande où tout propriétaire terrien peut exercer cette activité). Le critère d'appartenance à la population samie défini par le royaume de Suède au début du xx siècle a longtemps restreint celle-ci aux seuls éleveurs de rennes (Lundmark, 2007). Bien que réductrice et résultant d'une politique assimilationniste - puisque jusqu'à la colonisation du Nord de la Suède, la pêche et la chasse 
étaient des activités économiques aussi importantes que l'élevage pour les Samis - cette définition atteste du rôle central de l'élevage de renne dans l'identité samie.

Le renne et l'élevage occupent également une place centrale dans l'activité économique. Il y a entre 220000 et 280000 rennes pour environ 4600 propriétaires en Suède (60\% d'hommes et $40 \%$ de femmes), dont 2500 en tirent leur principal revenu d'après les statistiques du Parlement sami de Suède (Sametinget, 2013) et peuvent donc être considérés comme éleveurs à plein-temps. C'est aujourd'hui une activité économique orientée vers la production de viande. Ce sont principalement les jeunes animaux (faons mâles) qui sont vendus au début de l'hiver. Une part du troupeau sert également à l'autoconsommation puisque les mâles adultes sont abattus à cette fin en septembre avant le rut. L'élevage du renne n'est présent et autorisé que dans les régions « historiques » de la Laponie, c'est-à-dire dans le tiers Nord du pays (près de 200000 km²) où ne résident que $6,6 \%$ de la population suédoise (soit environ 3 habitants $/ \mathrm{km}^{2}$ ). À la fin du XIX ${ }^{e}$ siècle, ce territoire a été divisé en 51 communautés d'élevage par l'État suédois, aujourd'hui encore appelées sameby ${ }^{4}$ en suédois. Un sameby est la structure légale au sein de laquelle les éleveurs peuvent exercer l'activité d'élevage. Il regroupe plusieurs dizaines, voire centaines d'éleveurs, et se compose de sijdda ${ }^{5}$ (aussi orthographié siida en Sami du Nord), unité de base de l'organisation sociale traditionnelle des Samis, qui regroupe en hiver une ou deux familles nucléaires étroitement apparentées. Le sameby est aussi une entité territoriale au sein de laquelle les membres bénéficient du droit de pâturage libre ${ }^{6}$ quel que soit le statut foncier des terres (forêt privée ou public, parc national, terre agricole, etc.).

\section{Le cycle annuel de l'élevage}

Comme de nombreux systèmes d'élevage, celui du renne a connu de profondes transformations au cours du xx ${ }^{e}$ siècle (cf. Manker, 1954). La domestication du renne peut être caractérisée de labile. Selon les actions de l'éleveur, le renne peut devenir fortement domestiqué ou, en l'absence de contact avec l'homme, se comporter comme un animal sauvage. Jusqu'aux années 1910, l'élevage du renne pouvait être qualifié d'intensif. Les femelles étaient encore traites et les troupeaux étaient de petite taille, l'éleveur gardait, sur son troupeau un contrôle étroit et quotidien en toute saison. La fermeture de la frontière entre la Suède et la Norvège a entraîné une migration massive des Samis du Nord, qui 
ont importé leur modèle extensif vers le Sud de la Laponie, comme à Jokkmokk. Dans les années 1960, l'usage des animaux de bât et de trait, tout comme la pratique du ski, se sont raréfiés avec l'apparition des motoneiges. En conséquence, le troupeau de renne au début du xxI ${ }^{\mathrm{e}}$ siècle est moins domestique qu'il ne l'était un siècle plus tôt.

Lélevage du renne suit aujourd'hui un cycle annuel rythmé par deux transhumances, au printemps et en automne, entre les territoires d'été situés à l'Ouest du pays en direction de la frontière avec la Norvège et les territoires d'hiver dans les forêts de conifères proches du golfe de Botnie. Il s'agit donc d'une migration altitudinale entre la toundra comprise entre 700 et $2000 \mathrm{~m}$ et la taïga à plus basse altitude. La migration de printemps a lieu fin avril, juste avant la période de mise bas qui a lieu début mai, tandis que la migration d'hiver a lieu entre novembre et décembre, après le rut. Ce passage des territoires d'été aux territoires d'hiver est également le moment où le troupeau et les éleveurs d'une même communauté se divisent en structures plus petites, les sijdda. Chaque sijdda assure alors le gardiennage de son troupeau dont l'effectif peut aller de quelques centaines d'animaux à quelques milliers pour les plus grands dans la région de Jokkmokk. Ces deux migrations ne doivent pas pour autant occulter le fait que les troupeaux sont en déplacements constants. L'élevage de rennes est donc un élevage extensif où les animaux se déplacent d'Ouest en Est pendant l'hiver et le printemps, et d'Est en Ouest pendant l'été et l'automne.

\section{Des lichens et des rennes}

Pendant l'hiver, la principale source de nourriture pour les troupeaux de rennes est le lichen qui peut représenter jusqu’à $80 \%$ du régime hivernal de ces cervidés (Heggberget $\&$ al., 2002).

\footnotetext{
Le « lichen des rennes »

Les principales espèces

de Cladonia consommées

par les rennes en hiver sont

(A) Cladonia stellaris,

(B) Cladonia rangiferina

et (C) Cladonia arbuscula.
}

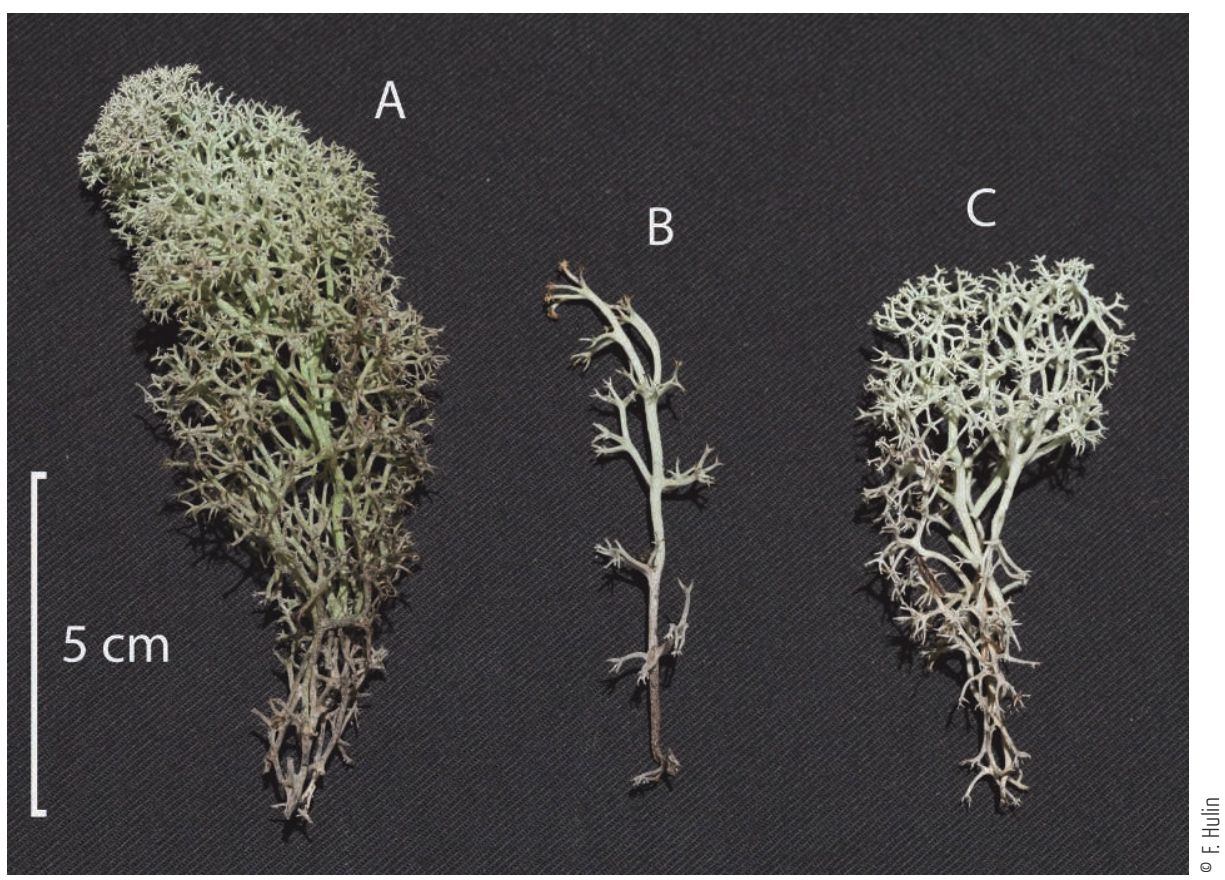




\section{Lexique des mots en sami de Luleå}

Båddne : définit le fond du manteau neigeux, la couche qui est en contact avec le sol et la végétation.

Båddne-vihke : «båddne-malade », décrit un état de pâturage mauvais lorsque le båddne s'est transformé en glace, rendant l'accès au lichen difficile pour les rennes.

Guohtom: bien que traduit par "pâturage ", guohtom signifie en hiver, outre la présence de lichen, la possibilité pour les rennes d'y accéder à travers le manteau neigeux.

Hiergge : renne mâle castré.

Sämolednam : "terre à mousse », végétation dominée par les mousses.

Sijdda : regroupe généralement une ou deux familles avec un lien de parenté étroit. Également appelée aujourd'hui " groupe d'hiver», la sijdda correspond unité de base de l'organisation sociale traditionnelle des Samis.

Tjuovvo : renne reconnu pour ses qualités de "suiveur », facile à conduire.

Vistasednam : " terre à lichen », végétation dominée par les lichens du genre Cladonia.

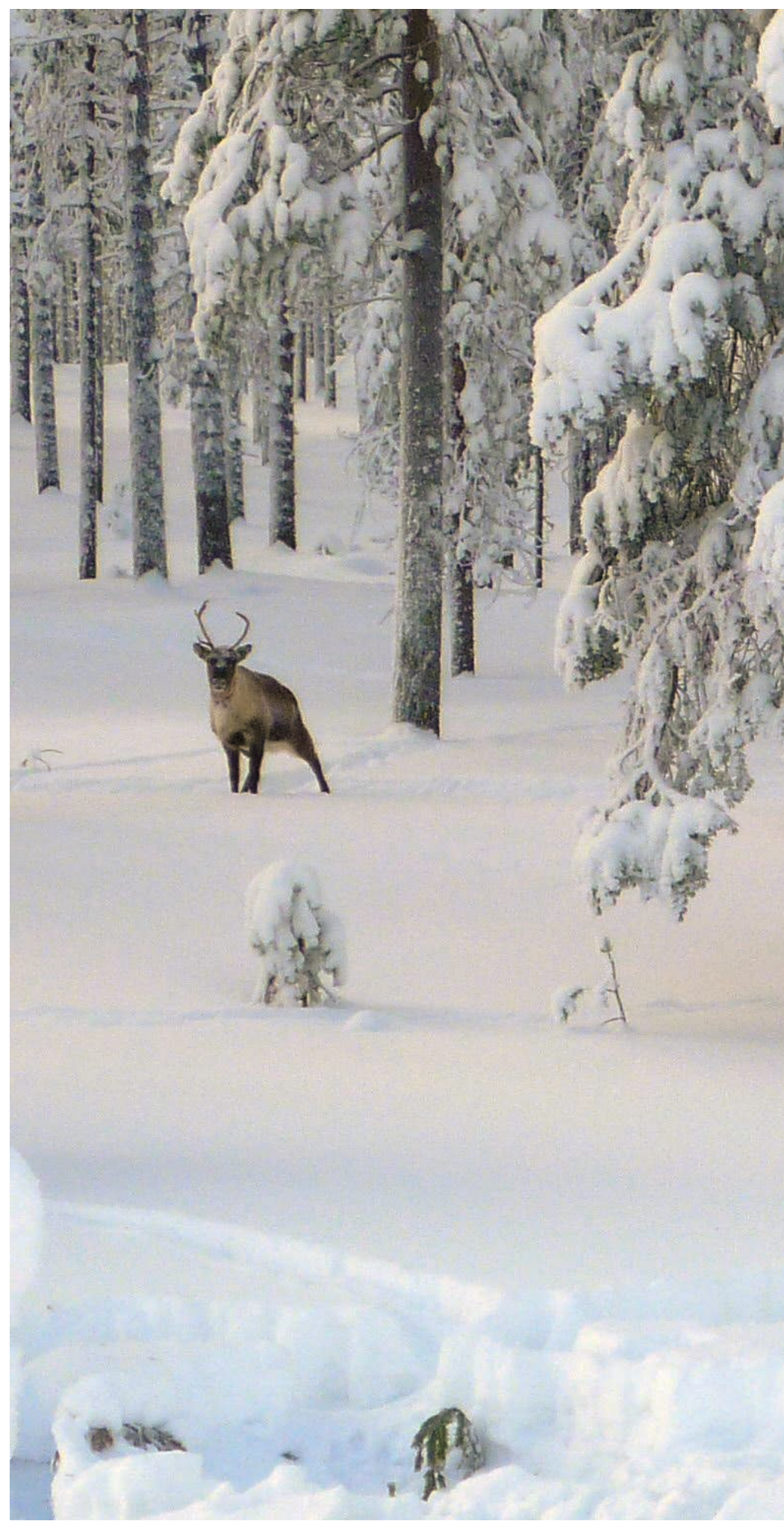




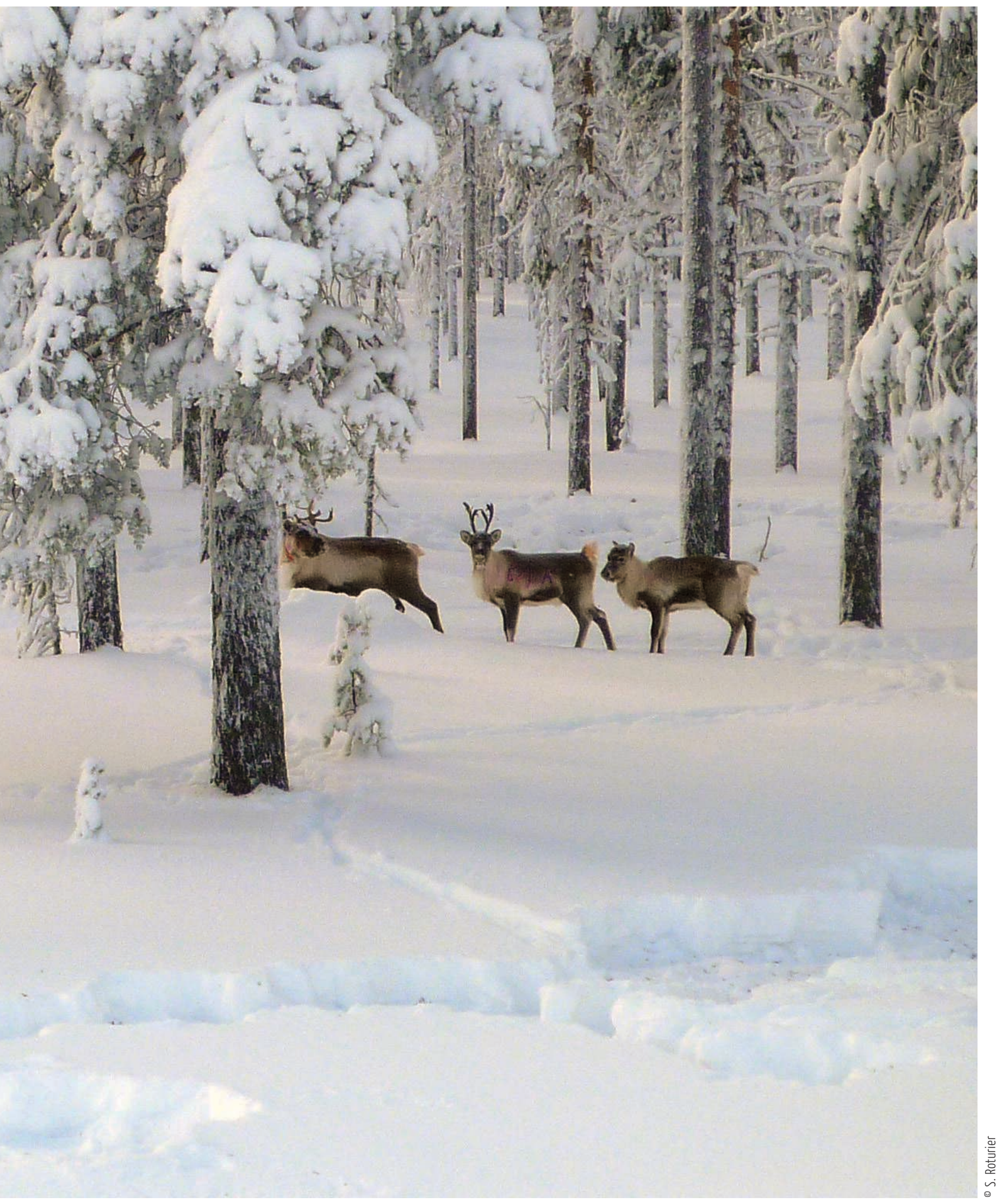


facteurs pris en compte par les éleveurs de rennes lorsqu'il s'agit de comprendre le pâturage. Lorsqu'ils l'observent, l'évaluent, en parlent, les éleveurs se concentrent d'abord et avant tout sur la neige. Dans la région de Jokkmokk, la moyenne annuelle des températures est de $-1{ }^{\circ} \mathrm{C}$, allant de $-14{ }^{\circ} \mathrm{C}$ en moyenne en janvier à $+14^{\circ} \mathrm{C}$ en juillet. La neige est présente entre 200 et 225 jours par an avec une épaisseur maximale de 70 à $90 \mathrm{~cm}$ (moyennes sur la période 1961-1990, SMHI), mais pouvant fortement varier en fonction des différences d'altitude. Le manteau neigeux est donc une composante essentielle qui permet ou non le pâturage. Deux mécanismes météorologiques peuvent compromettre le pâturage :

- des chutes de neige importantes peuvent entraîner la formation d'un manteau neigeux trop épais,

- un réchauffement de la température au-dessus de $0^{\circ} \mathrm{C}$, parfois accompagné

de pluie, suivi d'un refroidissement entraîne la formation d'une croûte de glace.

Ces phénomènes sont décrits sous les termes de «fonte-regel » et " pluie sur neige » dans la littérature scientifique (Putkonen \& Roe, 2003). Les conséquences peuvent alors être dramatiques pour les troupeaux puisque les animaux ne parviennent plus à creuser la neige et à s'alimenter.

\section{Le pâturage et la neige}

Les lichens du genre Cladonia consommés en quantité importante par les troupeaux de rennes représentent une source d'alimentation très digeste et à forte teneur énergétique par comparaison avec les plantes vasculaires (Storeheier $\&$ al., 2002). Bien que le lichen soit essentiel à la survie des rennes en hiver, les éleveurs ne le considèrent généralement

\section{Catégories samies et classification forestière Végétations forestières correspondant aux catégories sämolednam (gauche) et vistasednam (droite) pour les éleveurs, « type myrtille » et « type lichen » pour les forestiers en se basant sur un critère : la couverture de lichen au sol est-elle supérieure ou non à 50 \% .}
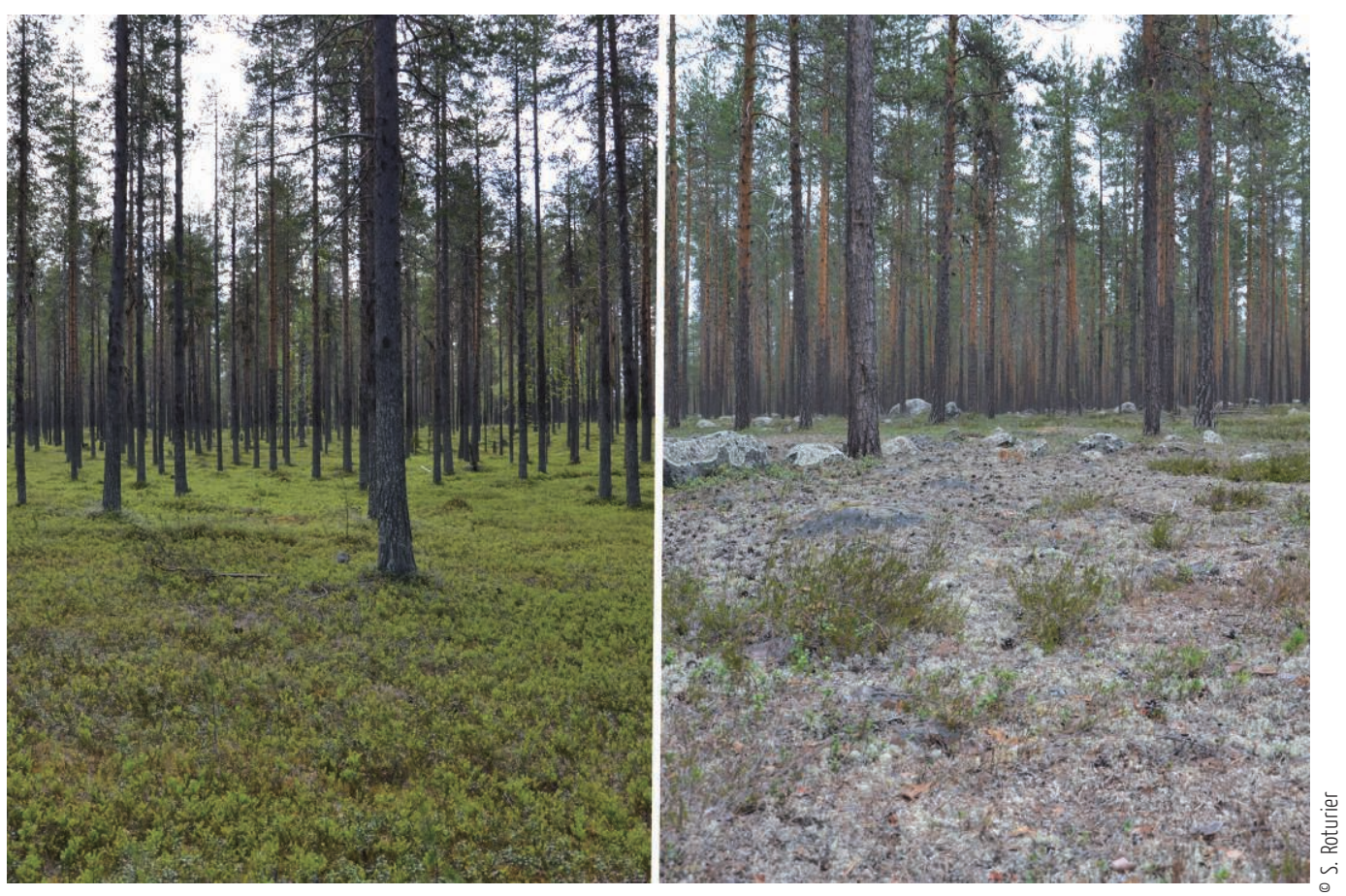
que comme l'un des trois facteurs nécessaires au pâturage, les autres facteurs étant l'état du båddne - c'est-à-dire le fond du manteau neigeux qui est en contact avec le sol et la végétation - et l'épaisseur du manteau neigeux. Les éleveurs considèrent que le pâturage est possible si et seulement si deux de ces facteurs sont réunis. Un éleveur expérimenté de 35 ans nous l'explique ainsi :

« On peut généralement dire que le pâturage dépend de trois choses : la neige, le båddne et le lichen. Si deux sur trois sont bons, alors le pâturage l'est aussi. Par exemple si le lichen est haut, qu'il n'y a pas beaucoup de neige, mais par contre de la glace [au niveau du båddne], ça peut marcher car le lichen dépasse au-dessus de la glace. Si le lichen est très petit, mais qu'il n'y a pas de glace et peu de neige, même si le lichen n'est pas bon, les rennes peuvent pâturer. Le pâturage, c'est toute une science en fait ${ }^{7} !$ ».

De tels raisonnements ne sont jamais explicités, encore moins lorsque les éleveurs parlent avec les forestiers et qu'ils utilisent le mot suédois bete « pâturage ». Ce mot désigne alors des choses différentes pour les Samis suivant le contexte comme le lichen, la neige, voire la combinaison des deux. Comme nous l'avons montré (Roturier \& Roué 2009), il ne s'agit pas là d'une confusion ou même d'une métonymie, mais d'une simplification du sens complexe que revêt le mot sami guohtom, "pâturage ", induite par la traduction en suédois. Le mot sami guohtom et surtout la définition qu’en donnent les éleveurs, permet d'aller au cœur de la représentation des pâturages hivernaux chez les Samis. En français comme en suédois, le mot pâturage est ambigu car il peut faire référence à un lieu, la pâture, autant qu'à l'action de pâturer, le pacage. La représentation samie du pâturage et l'utilisation du mot guohtom lèvent totalement cette ambiguité puisqu'il n'y a pâture que là et à partir du moment où les rennes peuvent pâturer :

« Guothom, veut dire, en fait, que le renne peut manger. C'est pourquoi ça inclut les conditions de neige, l'épaisseur de la neige, le sol, ce qu'il y a sur le sol, s'il y a du lichen. L'idée est que le renne puisse manger : guohtot. Le renne guohtot : le renne mange, le renne... pâture ».

Le pâturage dans le sens où les éleveurs samis se le représentent, ne se restreint donc pas à la communauté végétale pâturée par les troupeaux de rennes, c'est-à-dire au lichen terricole. Guohtom porte en lui la notion d'accessibilité à ce lichen à travers le manteau neigeux. Or dans des conditions climatiques d'une variabilité extrême, un pâturage peut être accessible un jour mais pas le lendemain. On comprend alors aisément qu'une forêt, bien qu'abondamment couverte de lichen, puisse ne pas être un bon pâturage, un guohtom, quand la neige restreint ou bloque complètement l'accès au lichen. On comprend également pourquoi la pluie peut « détruire » le pâturage quand elle conduit à la formation de glace sur la neige. Cette analyse invite à réexaminer les effets de la foresterie sur l'élevage en ne considérant plus uniquement le tapis de lichen, mais aussi le manteau neigeux (Roturier \& Roué, 2009).

\section{Analyse ethnoscientifique du pâturage}

Dans le champ de l'ethnoscience, guohtom est ce que C. Friedberg (1997) a appelé une catégorie complexe, c'est-à-dire une catégorie définie par des critères ou attributs appartenant à différents domaines, ici botanique, climatique, ou encore sylvicole. En adoptant 
cette démarche, c'est-à-dire en considérant les catégories autochtones et les critères sur lesquels se basent les éleveurs pour les définir, il est possible d'appréhender la discontinuité de la taïga telle qu'elle apparaît aux éleveurs samis et de percevoir ainsi la diversité du pâturage. C'est ce que nous allons voir à travers deux catégories sémantiques utilisées par les éleveurs de rennes pour décrire la composition de la strate herbacée et ses interactions avec le manteau neigeux, en les comparant aux catégories utilisées par les forestiers. Dans la région de Jokkmokk les éleveurs distinguent les vistasednam « terres à lichen », des sämolednam « terres à mousse ». La strate herbacée des premières est dominée par les lichens du genre Cladonia tandis que les secondes sont dominées par les mousses (principalement Pleurozium schreberi). Ces deux catégories se rapprochent de catégories utilisées par les forestiers dans leur classification de la fertilité des sols qui se base sur la composition de la strate herbacée et sur l'abondance du lichen pour définir les stations les moins fertiles (Hägglund \& Lundmark, 1982). Utilisée par toutes les compagnies forestières, cette classification est la base même de la culture forestière. La conduite d'un peuplement forestier, et donc les aménagements qui y seront faits, dépendent de cette classification.

Pour les éleveurs, lorsque les conditions de neige sont normales, le «bon pâturage » sera logiquement là où le recouvrement en lichen est le plus important, c'est-à-dire dans les vistasednam. Cependant la distinction faite par les Samis, qui ne s'arrête pas à l'abondance en lichen, prend toute son importance lors d'événements météorologiques extrêmes comme une fonte-regel décrit précédemment, phénomène se produisant presque tous les ans avec une intensité variable, mais de plus en plus fréquent en raison du réchauffement climatique (Johansson \& al., 2011). Pour les éleveurs, il importe avant tout que le båddne soit en bon état, c'est-à-dire que la neige ou la glace ne s'accroche pas à la végétation, ou ne la «verrouille » pas pour reprendre les termes employés par les éleveurs. Après des fonte-regel, le båddne peut être très différent suivant les types de végétation. D’après les éleveurs, les vistasednam où le lichen est le plus abondant, seront en effet très sensibles à une fonte-regel car l'eau issue de la neige fondue a propension à stagner sur le tapis de lichen, avant de regeler et d'encapsuler le tapis de lichen, souvent haut de seulement quelques centimètres, car régulièrement pâturé. C'est ce que les éleveurs appellent un båddne-vihke, un «båddne malade » (Ryd, 2001). À l'inverse, les sämolednam, les terres à mousses, seront moins sensibles au même événement car, selon les éleveurs, l'eau s'infiltre dans la mousse, laissant le lichen libre au-dessus de la glace qui vient se former sous le tapis de lichen. Dans ces conditions météorologiques particulières, le guohtom, la possibilité de se nourrir pour les rennes, se trouvera, non pas là où le lichen est le plus abondant comme en condition de neige normale, mais paradoxalement sur les terres où le lichen est plus rare, là où il reste accessible.

Comme le montre cet exemple relativement simple, ce ne sont pas les conditions météorologiques seules qui modifient les propriétés de la neige. La végétation influence également son métamorphisme. Les éleveurs samis sont experts dans la compréhension de ces phénomènes complexes ${ }^{8}$. Après leur dépôt au sol, les particules de neige sont modifiées par la température, l'accumulation ultérieure de neige, le vent, mais aussi des échanges de chaleur venant du sol ou des arbres. Ces facteurs dépendent à leur tour de la végétation au sol, mais aussi du couvert arboré (essences forestières, densité d'arbres à l'hectare, âge des peuplements ou encore forme des houppiers) qui affectent aussi bien la densité de la neige que l'accumulation ou la fonte du manteau neigeux (e.g. Winkler \& al., 2005). Se représenter les pâturages seulement à travers le lichen 
comme le font les forestiers ou les écologues conduit à des diagnostics erronés fondés sur un ethnocentrisme qui n'est pas l'apanage des gestionnaires et des scientifiques. Cette représentation unidimensionnelle du pâturage contraste avec celle, pluridimensionnelle et beaucoup plus riche des samis, qui prend en compte tous ces facteurs. En hiver la forêt boréale se présente aux éleveurs comme un paysage de neige extrêmement diversifié. Certains auteurs ont ainsi pu parler par métaphore de snowscape (Hellander \& Mustonen, 2004). Le pâturage devient une mosaïque aux dynamiques complexes, dont les caractéristiques varient non plus seulement dans l'espace mais aussi dans le temps.

\section{Savoirs et représentations samis}

\section{Contrôler le troupeau}

L'élevage de renne repose aujourd'hui sur un pâturage extensif où les animaux sont libres de se déplacer nuit et jour pour trouver eux-mêmes leur nourriture, au sein de territoires de plusieurs centaines de $\mathrm{km}^{2}$. Pour autant on ne saurait qualifier l'élevage de renne sami de ranching car en réalité, entre la migration d'automne et celle de printemps vers les terres de vêlage, les éleveurs cherchent constamment à maîtriser les déplacements de leur troupeau. Le pâturage joue alors un rôle primordial.

L'hiver est la période où l'accès à la ressource est la plus difficile pour les troupeaux, ce qui peut engendrer une surmortalité des jeunes et des avortements (Kojola \& al., 1995). La priorité des éleveurs de rennes est donc d'assurer la survie du troupeau pendant cette période. L'hiver est également la saison où le gardiennage est le plus intense. De décembre à mai, chaque sijdda doit maintenir son troupeau sur son territoire. Elle doit empêcher que ses animaux n'envahissent le territoire de la sijdda voisine, situé au mieux sur l'autre rive d'une rivière gelée, mais parfois seulement sur l'autre versant d'une colline. Les mélanges de rennes entre sijdda ne sont pas socialement acceptables : les animaux étrangers réduisent la ressource disponible pour le troupeau envahi et conduisent à une surcharge de travail pour trier les troupeaux. La concurrence avec les activités forestières et agricoles et le développement de nombreuses infrastructures (barrages, mines, routes, villes, etc.) sont également plus intenses sur les territoires d'hiver et accroît les risques de perdre des animaux. Les rennes égarés deviennent plus vulnérables face aux nombreux prédateurs (ours, loups, lynx, gloutons) et peuvent mettre bas en dehors des territoires de vêlage. Ce dernier aspect est extrêmement important car l'éleveur ne dispose que de quelques mois pour marquer ses faons ${ }^{9}$ et ainsi assurer le renouvellement de son troupeau. Contrôler le troupeau est donc primordial et le pâturage est le meilleur moyen pour l'assurer.

Les théories développées en écologie comportementale sur les stratégies de recherche de nourriture chez les grands herbivores sauvages mettent en rapport le temps passé par un individu sur un site avec la quantité et la qualité du pâturage (Senft \& al., 1987). Le manteau neigeux influence donc directement les déplacements des rennes. Lorsque les conditions d'accès au lichen sont bonnes, le troupeau reste groupé et ne se disperse pas. L'activité de gardiennage en est facilitée. Inversement, lorsque l'accès au lichen à 
travers la neige diminue, la capacité de charge diminue elle aussi (c'est-à-dire le nombre d'animaux pouvant subvenir à leur besoin alimentaire pour une surface donnée), et le troupeau doit se disperser sur une plus grande surface pour s'alimenter :

« Moins il y a à pâturer, plus les rennes bougent, cherchent à manger, c'est naturel. Pour rester, ils doivent trouver un bon pâturage. Quand les conditions de pâturage sont bonnes, on n'a pas besoin de sortir autant, et peut être même pas tous les jours. C'est comme ça! Plus le pâturage est mauvais, plus c'est du travail pour les garder ensemble et surveiller qu'ils aient quelque chose à pâturer ».

Lors d'aléas climatiques extrêmes, comme la formation d'une croûte de glace sur le manteau neigeux, les rennes se dispersent d'autant plus facilement et plus rapidement qu'ils ne s'enfoncent plus dans la neige. Le risque est alors grand pour les éleveurs de voir leurs rennes se disperser « comme des haricots secs sur le carrelage », tel qu'ils l'expriment souvent de façon imagée. Des expressions comme « se disperser », « décrocher » - sous-entendu du pâturage - ou au contraire « rester tranquille », sont extrêmement courantes lorsqu'il s'agit de décrire l'activité du troupeau, indiquant simultanément l'état du pâturage et le mouvement des rennes. Le pâturage est vu comme un enclos invisible, fragile certes, mais à l'intérieur duquel l'éleveur garde le contrôle du troupeau :

«Si on imagine les rennes comme des billes, et le pâturage comme des trous dans le parquet ${ }^{10}$. Les rennes font comme les billes, ils vont dans les trous. C'est comme ça qu'on les retrouve ».

plateau d'Ultevis cherchent

les rennes aux jumelles avant la migration d'automne.

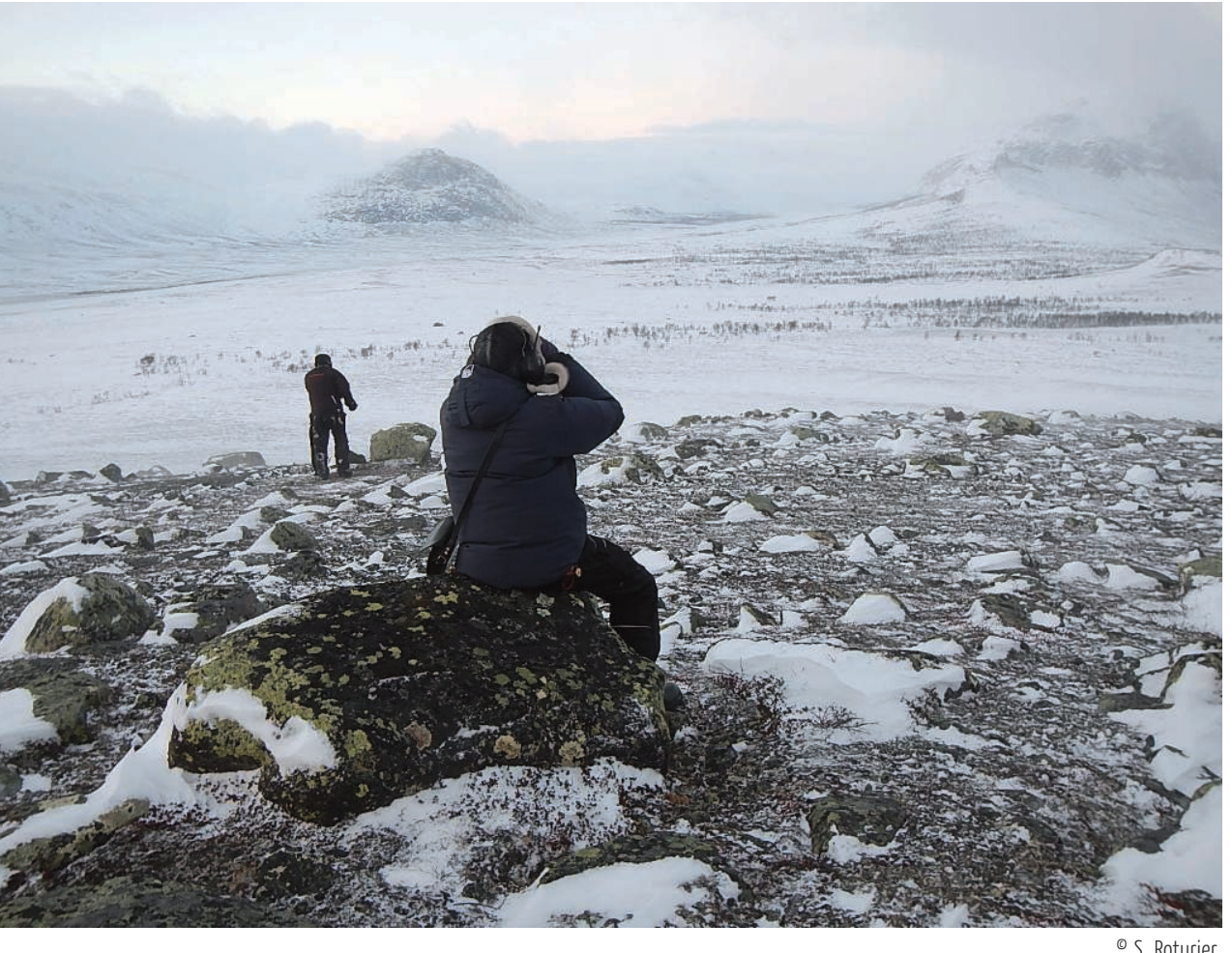

\section{Penser comme un renne}

Est-ce le renne qui suit le Sami ou au contraire les Samis qui suivent les rennes? Le renne estil domestique ou sauvage? Ce sont des questions souvent posées et, comme toutes les questions mal posées, jamais résolues. Comme nous l'avons expliqué, le renne n'est ni complètement sauvage, ni complètement domestique. En réalité, il existe un gradient de domestication au sein du troupeau, avec des animaux très fortement domestiqués pouvant être menés à la longe, les hiergge (mâles castrés), d'autres plus expérimentés à qui l'on met parfois des cloches, les tjuovvo, et d'autres enfin impossibles à approcher. Cela dépend aussi des circonstances avec une alternance d'interventions directes 


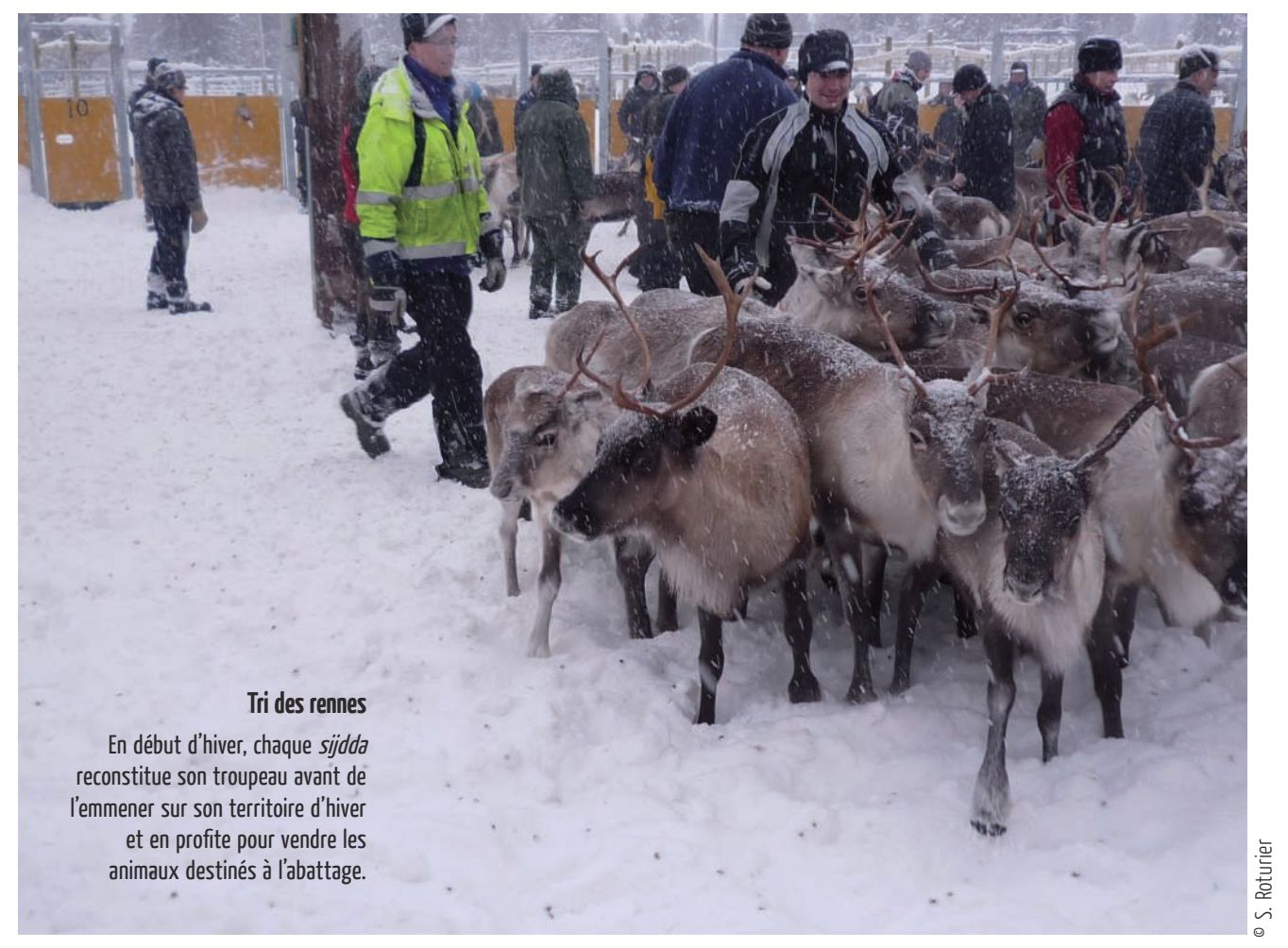

sur les animaux (tri, abattage, sélection, vaccination, marquage des faons) et de périodes pendant lesquelles le gardiennage est très souple. On laisse par exemple les femelles vêler en surveillant de loin, on n'intervient pas au moment du rut quand les mâles s'affrontent pour constituer leur harem et fertiliser les femelles. Par contre on est très proche des hiergge ou même de l'ensemble du troupeau dans certaines situations. On peut parler à ses rennes pour leur communiquer les décisions qu'on vient de prendre, pour, disent les éleveurs, qu'ils les comprennent ou encore s'adresser à un renne pour qu'il prenne la tête du troupeau quand un obstacle est difficile à franchir et qu'on a besoin de son aide pour y parvenir.

Rennes et éleveurs s'accordent sur le temps et le lieu de pâturage, mais dans les moments difficiles l'éleveur prend des décisions. Il essaye par exemple de retarder autant qu'il le peut l'éparpillement de ses rennes ou leur avancée vers les montagnes au printemps, mais quand il juge que le pâturage n'est plus bon, il déplace le troupeau, ou plutôt le laisse se déplacer dans la direction qu'il juge stratégique. Les éleveurs modèrent les mouvements du troupeau, le contrôlent sans excès, mais ne prennent une décision qu'après avoir examiné le comportement des rennes : « On ne sait jamais, mais les rennes savent. Grâce à notre savoir, on ne creuse pas au mauvais endroit. On sait. La terre nous montre où aller, quelle terre est utile, et les rennes nous montrent que l'on a fait le bon choix $»$. De fait, les rennes sont en accord avec les décisions des éleveurs, puisque ces derniers ne visent rien d'autre que de s'adapter aux circonstances en les analysant, en se mettant même à la place du renne. Cet ancien explique ainsi : "Quand je ne suis pas sûr du lichen, en général je le mets dans ma bouche et je sens s'il y a de la glace. Parce que dans la bouche, tu le sens s'il y a des 
cristaux de glace dans le lichen ». En fait, toute la science de l'éleveur consiste à « penser comme un renne », pour paraphraser A. Leopold, précurseur du mouvement écologiste, qui définissait ainsi l'éthique de la terre (la land ethic) : "apprendre à penser comme une montagne » (Leopold, 1949).

\section{$\&$}

Pour les Samis éleveurs de rennes, le pâturage est une science. Laissant leurs rennes pâturer le lichen en liberté au sein de vastes territoires forestiers, les Samis ont pleinement conscience que le pâturage n'est pas une réalité intemporelle. La représentation qu'ils s'en font s'oppose à une définition essentialiste du pâturage hivernal. Sous le climat boréal, dans la taïga, l'accessibilité au lichen sous le manteau de neige est en constante évolution. Ainsi le pâturage hivernal se construit et se détruit suivant les chutes de neige, les changements de température et la végétation. Pour les éleveurs, comprendre et maîtriser les interactions écologiques, physico-chimiques et météorologiques qui font et défont le pâturage, mobilise des savoirs de nature extrêmement variée et des années d'expérience (Alvarez de Torres, 2015). Enfin le pâturage n'est pas seulement une technique qui permettrait d'utiliser au mieux l'accessibilité au lichen pour guider et contrôler le troupeau à travers un parcours. C'est une situation où les besoins des animaux et la stratégie que les éleveurs mettent en place concordent en un même lieu. 


\section{NOTES}

Remerciements: Nous remercions chaleureusement les éleveurs de rennes qui nous recoivent avec patience depuis plusieurs années, notamment la famille Åstot. Ce travail a bénéficié du soutien du programme ANR BRISK (ANR-12_SENV-0005)

Photo d'ouverture: L. Åstot, 70 ans, éleveur respecté dans la communauté de Sirges, Jokkmokk. «Lorsque la neige fond, l'eau s'infiltre dans la mousse et la glace se forme sous le lichen». (Photo : S. Roturier).

1. Aux termes de Lapon et de Laponie se sont progressivement substitués ceux d'origine lapone de Sami (parfois orthographié Saami ou Sâme) et Sápmi. Ne ressemblant en rien au terme par lequel ils se désignent dans leur propre langue, les mots construits avec le radical lapp comportent également une connotation péjorative dans les pays scandinaves. Nous utiliserons donc le terme de Sami, en revanche nous conserverons celui de Laponie qui présente l'avantage d'être compris de tous sans véhiculer l'image discriminatrice propre au contexte scandinave.

2. La restauration écologique est le processus intentionnel qui vise à assister l'autoréparation d'un écosystème dégradé ou détruit.

3. L'hiver sera ici considéré comme la période de l'année où le manteau de neige est présent, c'est-à-dire environ de novembre à avril.

4. Sameby a longtemps été mal traduit par sami village, notamment dans la littérature anglophone.

5. Notons néanmoins que la loi n'autorise la présence des troupeaux sur les territoires d'hiver qu'entre le ler octobre et le 30 avril. D'autre part, ce droit est localement contesté par des groupes de propriétaires terriens aux marges de la Laponie. En 1996 un procès dans le Härjedalen a conduit à l'interdiction de pâturage dans des forêts privées. En 2011 en revanche, le procès dit « de Nordmaling » a renforcé le droit de pâturage des communautés samies le long du golfe de Botnie.
6. Les mots samis sont écrits en sami de Luleå, langue historique de la région de Jokkmokk. Bien que le sami du Nord soit aujourd'hui dominant en Laponie, plusieurs dialectes samis sont aujourd'hui encore utilisés.

7. Les citations sont issues d'interviews réalisées en suédois et traduites par les auteurs.

8. Un film de 3 minutes est accessible dans la version en ligne de cet article. Elle montre un éleveur expliquant au début de l'hiver, les phénomènes météorologiques et physiques qui ont conduit à la formation, puis à la fonte progressive d'une couche de glace sur le tapis de lichen.

9. Le marquage des faons nouveau-nés a lieu en juillet. C'est l'événement le plus important dans l'année pour l'éleveur et sa famille. En effet, un faon non marqué est perdu pour l'éleveur, et sera vendu par la communauté qui le trouvera. Les faons, alors âgés de 2 mois, suivent encore à cette période leur mère L'éleveur peut donc reconnaître son faon avant de l'attraper, et de le marquer par un découpage spécifique des oreilles. Chaque éleveur se voit attribuer une marque individuelle de propriété dès l'enfance, connue de tous et enregistrée légalement.

10. Faut-il préciser que cette explication nous a été livrée dans un sauna?

\section{POUR CITER CET ARTICLE}

Roturier, S. \& Roué, M. 2015 Le Pâturage c'est toute une science ! In Dupré, L., Lasseur, J. \& R. Poccard-Chapuis, Pâturages Techniques E Culture 63: 92-109. 


\section{RÉFÉRENCES}

Delaporte, Y. \& M. M. Roué 1986 Une communauté d'éleveurs de rennes. Vie sociale des Lapons de Kautokeino. Paris: Institut d'Ethnologie, Musée de l'Homme.

Esseen, P.-A., et al. 1997 Boreal forests, Ecological Bulletins 46: 16-47.

Friedberg, C. 1997 Diversité, ordre et unité du vivant dans les savoirs populaires, Natures Sciences Sociétés 5: 5-17.

Hägglund, B. \& J.-E. Lundmark 1982 Bonitering, Del 3. Markvegetationstyper - Skogsmarkflora. Jönköping: Skogsstyrelsen. [Également en ligne] URL: http://www-markinfo.slu.se/sve/veg/vegtyp.html. Consulté le 17 mai 2014.

Heggberget, T. M., E. Gaare \& J. P. Ball 2002 Reindeer (Rangifer tarandus) and climate change: importance of winter forage, Rangifer $22: 13-31$.

Hellander, E \& T. Mustonen 2004 Snowscapes, Dreamscapes: Snowchange Book on Community Voices of Change. Tampere: Tampere Polytechnic.

Ingold, T. 1976 The Skolt Lapps Today. Cambridge: Cambridge University Press.

Johansson, C., \& al. 2001 Multi-decadal changes in snow characteristics in sub-arctic Sweden, Ambio 40: $566-$ 574.

Kojola, I., \& al. 1995 Effects of lichen biomass on winter diet, body mass and reproduction of semidomesticated reindeer Rangifer t. tarandus in Finland, Wildlife Biology 1 : 33-38.

Leopold, A. 2000 [1949] Almanach d'un comté des sables. Paris: Flammarion.

Lundmark, L. 2007 Reindeer pastoralism in Sweden 1550 - 1950, Rangifer Report 12 : 9-16.

Manker, E. 1954 Les Lapons des montagnes suédoises. Paris: Gallimard, Géographie humaine 24.

Paine, R. 1964 Herding and husbandry: two basic concepts in the analysis of reindeer herding management, Folk 6: 83-88.

Putkonen, J. \& G. Roe 2003 Rain-on-snow events impact soil temperatures and affect ungulate survival, Geophysical research letters 30: 1188.

Roturier, S. 2009 Managing Reindeer Lichen during Forest Regeneration Procedures : Linking Sami Herders'Knowledge and Forestry. Umeå: Acta Universitatis Agriculturae Sueciae. (Thèse de doctorat SLU - MNHN).

Roturier, S. \& M. Roué 2009 Of Forest, snow and lichen: Sami reindeer herders'knowledge of winter pastures in northern Sweden, Forest Ecology and Management 258: 1960-1967.

Roué, M. 2012 Forêts boréales et gestion durable. Peuples autochtones, environnementalistes et forestiers. In S. Poirot-Delpech \& L. Raineau (Dir.) Pour une socio-anthropologie de l'environnement. Tome I Par-delà le local et le global. Paris: L'Harmattan.

Ryd, Y. 2001 Snö. En renskötare berättar. Stockholm: Ordfront.

Sametinget 2013 Renägare nyckeltal 2013 Svenska Sápmi. [En ligne] URL: http://www.sametinget.se/statistik/ renägare. Consulté le 17 mai 2014.

Senft, R. L., et al. 1987 «Large herbivore foraging and ecological hierarchies, BioScience 37: 789-799.

SMHI 2009 Sveriges meteorlogiska och hydrologiska institut. [En ligne] URL: http://www.smhi.se. Consulté en janvier 2009.

Storeheier, P. V., \& al. 2002 Nutritive value of terricolous lichens for reindeer in winter, The Lichenologist 34: 247-257.

Torres Alvarez de, M. -F. 2015 Prairie naturelle, Campero et ingénieur. In Dupré, L., Lasseur, J. \& R. Poccard-Chapuis (Dir.) Pâturages, Techniques \& Culture 63: 74-91

Winkler, R. D., Spittlehouse, D. L. \& Golding, D. L. 2005 Measured differences in snow accumulation and melt among clearcut, juvenile, and mature forests in southern British Columbia, Hydrological Processes $19: 51-62$. 


\section{RÉSUMÉ}

«Le pâturage, c'est toute une science! " Savoirs écologiques sur la neige et représentation samie du pâturage. En Laponie suédoise, les Samis éleveurs de rennes doivent aujourd'hui partager leur territoire avec l'industrie forestière. Le conflit qui en résulte a, jusqu'à présent, porté l'attention sur les lichens, qui constituent l'alimentation principale des troupeaux pendant les 5 mois de l'hiver boréal. Notre analyse montre que ni la présence ni l'abondance du lichen ne suffisent pourtant à définir le pâturage tel que les éleveurs se le représentent. La catégorie sémantique samie, guohtom, définit le pâturage, non pas de manière essentialiste, mais par rapport à son accessibilité à un moment donné. En prenant en compte l'accès au lichen à travers le manteau neigeux, la science des éleveurs consiste à analyser les nombreuses interactions entre la végétation, la neige, le climat et le milieu qui produisent, ou détruisent du jour ou lendemain, le pâturage. Ces savoirs écologiques permettent aux éleveurs samis de contrôler et guider les rennes à travers la taïga. Tout au long de l'hiver, ils évaluent les changements d'état du pâturage, anticipent les déplacements de leurs rennes et élaborent des stratégies d'utilisation du territoire. La science du pâturage émane aussi de la capacité des éleveurs à penser comme un renne. Plus qu'une expertise écologique et technique, le pâturage devient alors un état d'équilibre, dans l'espace et dans le temps, où la volonté des éleveurs s'harmonise avec les besoins des animaux.

\section{ABSTRACT}

«Pasture, a science in its own right! » Ecological knowledge about snow and the Sami representation of grazing. In Swedish Lapland, Sami reindeer breeders must now share their territory with the forest industry. Until recently, the resulting conflict was focused on lichens, which constitute the main food source for the reindeer herds during the five months of northern winter. Our analysis shows that neither the presence nor the abundance of lichen is sufficient to define pasture as represented by the herders. The Sami semantic category, guohtom, describes pasture not in its essence, but in relation to its accessibility at the time that the speaker expresses himself. By taking the access to lichen through the snow cover into account, the herders' science consists of analyzing the many interactions between the vegetation, the snow, the climate and the environment that produces or destroys the pasture from one day to the next. This ecological knowledge allows Sami herders to control and guide the reindeer over the taiga. Throughout the winter, they assess the changes in the state of the pasture, plan the movement of their reindeer and develop land-use strategies. Pasture science is also the outcome of the ability of the herders to think like reindeer. More than ecological and technical know-how, the pasture then becomes a state of balance in space and in time, where the desires of the breeders are synchronized with the needs of the animals.

\section{MOTS CLÉS}

ethnoscience, lichen, neige, Sami, savoir écologique traditionnel

\section{KEYWORDS}

ethnoscience, lichen, snow, Sami, traditional ecological knowledge 